\title{
EMPLEO DEL AGUA DE MAR CON SANGUAZA EN LA PRODUCCIÓN DE BIOMASA MICROALGAS DE NANNOCHLOROPOSIS OCEANICA EN CONDICIONES DE LABORATORIO
}

\author{
Using Seawater with Sanguaza in the Production Biomass Microalgas \\ of Nannochloropsis Oceanic Under Laboratory Conditions.
}

Luis Alberto Huayna Dueñas ${ }^{1}$, José Luis Romero Bozzetta ${ }^{1}$, William Guzmán Sanchez ${ }^{1}$, Adelfio Basilio A. ${ }^{2}$

\begin{abstract}
RESUMEN
El objetivo de la investigación fue determinar el efecto que produce la aplicación del agua de mar con sanguaza en la producción de biomasa micro alga de Nannochloropsisoceanica en condiciones de laboratorio. en la metodología se realizo una investigación explicativa experimental. La población estuvo conformada por la microalga clorofila Nannochloropsisoceanica. La muestra fueron alícuotas del cultivo de Nannochloropsisoceanica tomadas diariamente por un tiempo de 10 días, a las cuales se les evaluó pH, temperatura y conteo celular. Nos dio como resultado las concentraciones del medio heterotrófico sanguaza/agua de mar 5,0\% y $10 \%$ v/v los cuales presentaron el mayor número de células $/ \mathrm{ml}$ producidas en 144 horas de crecimiento. En conclusión se demuestra la potencialidad de uso del medio heterotrófico sanguaza/agua de mar en estudios escalables a nivel piloto de producción de biomasa micro alga de Nannochloropsisoceanica.
\end{abstract}

Palabras clave: Cinética de Crecimiento, Nannochloroposisoceanica, Sanguaza, Fotobiorreactor.

\begin{abstract}
The objective of the research was to determine the effect produced by the application of seawater with sanguaza in the production of microalgae biomass of Nannochloropsisoceanica under laboratory conditions. In the methodology, an experimental explanatory research was carried out. The population consisted of the microalga chlorofila Nannochloropsisoceanica. The samples were aliquots of the Nannochloropsisoceanica culture taken daily for a period of 10 days, at which $\mathrm{pH}$, temperature and cell count were evaluated. We obtained as a result the concentrations of the heterotrophic sanguaza / seawater $5.0 \%$ and $10 \% \mathrm{v} / \mathrm{v}$ which presented the highest number of cells / ml produced in 144 hours of growth. In conclusion, the potential use of the heterotrophic sanguaza / seawater in pilot scale studies of microalgae biomass production of Nannochloropsisoceanica is demonstrated.
\end{abstract}

Keywords: Growth kinetics, nannochloroposisoceanica, sanguaza, photobioreactor

${ }_{1}^{1}$ Docente de la Facultad de Ciencias, Universidad Nacional José Faustino Sánchez Carrión (Lima - Perú). Email: vinvestigacion@unjfsc.edu.pe 


\section{INTRODUCCIÓN}

En un mundo donde los requerimientos económicos para la expansión cambian rápidamente, el petróleo se encuentra en el centro de estos cambios; para países como China y otros, las fuentes del petróleo son el factor dominante en sus economías (Song et al. 2008).

Debido al agotamiento de las reservas de petróleo y a las consecuencias ambientales del uso excesivo de los combustibles fósiles, la producción de combustibles alternativos posee gran atención (Ma y Hanna 1999; Chisti 2007; Song et al. 2008; Meng et al. 2008; Kalia\&Purohit 2008). Actualmente las fuentes existentes de energía son: energía termonuclear, solar, eólica, geotérmica (Veziroglu y Barbir 1992).

De entre los reemplazos a los combustibles fósiles, el Biodiesel es uno de los más importantes (Ma y Hanna 1999), ya que puede ser producido a partir de aceites vegetales, grasa de cocina reciclada, aceites de desecho, grasa animal y de lípidos provenientes de microalgas (Van Gerpen 2005; Song et al. 2008).

Las algas (macro y microalgas) han sido utilizadas por centurias por diferentes civilizaciones en la alimentación humana y animal. Las microalgas o algas microscópicas, vienen siendo cultivadas de manera industrial para la producción de proteínas, vitaminas y otros suplementos nutritivos; son empleadas en simbiosis con las bacterias para la remoción de fosfatos y nitratos de aguas residuales, como biofertilizantes (Mora et al., 2005). Su cultivo se ha visto incrementado por ser fuente de ácidos grasos insaturados, minerales, pigmentos, enzimas, aceites esenciales, antibióticos y otros metabolitos biológicamente activos (Bermejo et al., 2002).

Algunos autores han mencionado que las diatomeas (Bacillariophyta), los dinoflagelados (Dinoflagellata), las Haptophyta y las algas verdes (Chlorophyta) podrían contar aproximadamente por el 40, 10 y $6 \%$ de las especies de eucariotas presentes en el fitoplancton marino actual, mientras que la suma de otros grupos contribuiría un $2 \%$ (Simon et al., 2009).

Las microalgas son organismos unicelulares que pueden ser cultivadas muy rápidamente (Chisti 2008), a diferencia de las plantas poseen una estructura unicelular sin tallos, hojas o raíces permite que la extracción de los productos sea más fácil (Avagyan 2008); del cultivo de estas, la obtención de biomasa es el principal objetivo, ya que esta es la forma más común de energía renovable, ya que posee un gran potencial para resolver problemas como el agotamiento de los recursos no renovables (Miao y Wu 2004; Li et al. 2008).

Las algas microscópicas han merecido considerable atención por su potencialidad como fuente de biodiesel, por la posibilidad de suplir de manera parcial la escasez de suministro de petróleo y asimismo, se presentan como una alternativa a los precios elevados de las materias primas tradicionales, para la obtención de los biocombustibles como son la soja, caña de azúcar, maíz, palma aceitera, colza, grasas animales, residuos de grasas, entre los más representativos (Knothet al., 1997; Lee, 1997; Garibay et al., 2009; Rodolfi et al., 2009).

El uso de microalgas para la producción de biomasa y producción de biodiesel es una alternativa ventajosa debido al elevado contenido de lípidos que acumulan, elevada eficiencia fotosintética, su capacidad de crecer tanto en aguas marinas, dulces, residuales y salobres, así como a su alta velocidad de crecimiento (Garibay et al., 2009; Xiaoling et al., 2006).

Para el incremento en la obtención de lípidos el sistema de cultivo debe poseer ciertas condiciones, entre estas que el medio sea rico en nutrientes (Paerl et al. 2001; Sellner et al. 2003; Liu et al.2008; Schenk et al. 2008), además que exista una buena transferencia de la luz (Sastre et al. 2007), dinámica del medio (Barbosa 2003), tamaño de burbuja y su distribución dentro del sistema (Barbosa 2004), intercambio gaseoso (Eriksen et al. 2008) y la transferencia de masa (Molina Grima et al. 1999).

Se reporta que reunir solo la mitad de biodiesel que necesita el transporte existente en EE.UU, requiere de grandes áreas de cultivo para producir mayor cantidad de oleaginosas. En el caso de palma aceitera (Elaeisguineensis) y jatropha (Jatrophapodagrica), plantas de buen rendimiento, necesitarían que el $24 \%$ y $45 \%$ del total de tierra respectivamente, esté dedicado a su cultivo para cubrir solamente $50 \%$ de la necesidad de combustible de transporte.

Es evidente que en un futuro inmediato la contribución de los cultivos de oleaginosas no alcanzará para reemplazar los combustibles líquidos, por lo que algunas compañías están intentando comercializar biodiesel a partir de la biomasa algal obtenida en cultivos (Knotheet al., 1997; Lee, 1997).

Si se lograría implementar el uso de microalgas para producir biodiesel, entre 1 y $3 \%$ del total de área de cultivo de los EE.UU., serían suficientes para producir la biomasa que satisfaga el $50 \%$ del combustible que necesita el transporte (Sheehan et al., 1998) y su cultivo no comprometería la producción de alimento, forraje y otros productos.

Dependiendo de las especies, las microalgas producen diferente tipos de lípidos, hidrocarburos y otros aceites complejos (Álvarez y Zarco, 1989; Guschina y Harwood, 2006), de los cuales no todos son adecuados para producir biodiesel.

La cantidad total de lípidos, así como la tipología de los ácidos grasos presentes, además de ser específica para cada especie, está ligada a factores ambientales como la intensidad luminosa, $\mathrm{pH}$, salinidad, temperatura, concentración de nitrógeno y otros nutrientes en el medio de cultivo (Álvarez, 1989).

Arias et al. (2013) e Hirata et al. (2001) señalaron que entre las microalgas de potencial importancia para la producción de biocombustibles (biodiesel) que existen en los cuerpos de agua marina, se encuentran (en base seca, tienen lípidos): Chlorellaminutissima (57\%), Chlorellavulgaris (57\%), Nannochlorissp. (40\%), Nannochloropsisoculata $(8 \%-54 \%)$, Nannochloropsissp. (24\% - 60\%) y

Tetraselmissuecica(20\% - 54\%).

Para la producción de biomasa algal marina se pueden emplear: cultivo autotrófico pudiendo utilizar diferentes fuentes nitrogenadas como urea, fosfato di amónico, nitratos, nitritos, etc; y en cultivo heterotrófico como ensilado de pescado puede metabolizar diversos 
aminoácidos y vitaminas, sintetizar ácidos grasos poli insaturados (Bold y Wynne, 1985).

Actualmente se realizan esfuerzos para la generación de nuevas metodologías ydesarrollo tecnológico para la producción masiva de microalgas y sus derivados, mediante el uso de medios de cultivos alternativos. Una alternativa a los medios de cultivo tradicionales ya estandarizados (puros y químicamente definidos, como el medio Guillard) son los sustratos orgánicos, mayormente subproductos de otras industrias y en cuya composición se encuentran fuentes de C, N, P, microelementos y oligoelementos. Utilizándose en la actualidad diversas harinas (de pescado, soya, lombriz de tierra, girasol), gallinaza, gas, aceites, melaza de caña, exudados gomosos y residuos pesqueros como la sanguaza (Herrero et al., 1994).

En Perú, los residuos pesqueros se producen en grandes cantidades como consecuencia de la intensa actividad de la industria pesquera, que es una de las actividades extractivas importantes del país. Los residuos como vísceras, cabezas, piel, huesos y colas, se cuantifican alrededor del $50 \%$ de la materia prima, resultando finalmente en contaminantes al ser vertidos indiscriminadamente al medio ambiente, provocando problemas de contaminación y enfermedades.

Un residuo de importancia es la "sanguaza", efluente constituido por agua, sangre y sólidos de pescado, generado durante su almacenamiento en pozas y transporte hacia los cocinadores en las fábricas de pescado. Sin embargo, estos residuos pueden ser aprovechados eficientemente si reciben un buen tratamiento para transformarlos (Gonzales y Marín, 2005).

En nuestro medio existen industrias que generan fuentes nitrogenadas residuales producto de su actividad, especialmente la industria de la harina de pescado, la cual produce líquidos residuales tales como: "agua de cola", "agua de bombeo" y "sanguaza". La utilización de la sanguaza constituye una alternativa de tecnología limpia de los ambientes pesqueros, pues una tonelada métrica de pescado procesado produce 50 litros de sanguaza y a nivel nacional se han generado más de 500 millones de litros, representando un verdadero problema en las costas del Perú. Por lo tanto, su uso como medio de producción de biomasa algal evitaría la contaminación de puertos.La "sanguaza" contiene cantidades apreciables de proteínas $(6,3 \%)$, grasas $(3,2 \%)$, sólidos solubles e insolubles $(3,5 \%)$, sales minerales $(1,8 \%)$ y otros, las cuales podrían ser aprovechadas para la producción de la biomasa algal (Blas et al., 2011).

El Perú como país que se encuentra en la orilla del Océano Pacífico y además poseedor de una fuerte industria pesquera que elimina un buen volumen de "sanguaza", esta se podría utilizar en un futuro para la propagación de biomasa algal oleaginosa para la producción de biodiesel (biocarburante), además se podrían obtener otros subproductos como la glicerina y producir otros productos a partir de la biomasa algal como alcohol y proteína unicelular. En el Perú se vienen realizando trabajos de investigación en la producción de biodiesel a partir de microalgas en institutos de investigación como IMARPE y en universidades como Universidad Nacional de Trujillo, Universidad Nacional del Santa y otras. A nivel mundial países como Chile, Ecuador, Colombia, Brasil, México, Costa Rica, Estados Unidos de Norteamérica, España y otros, ya tienen implementados plantas pilotos e industrias de producción de biodiesel.

Determinar el efecto que produce la aplicación del agua de mar con sanguaza en la producción de biomasa microalgal de Nannochloropsisoceanica en condiciones de laboratorio.

\section{MATERIALES Y MÉTODOS}

La población estuvo conformada por la micro alga clorofita Nannochlropsisoceanica que fue obtenida del Laboratorio larval de camarones de la Facultad de Ingeniería Pesquera de la UNJFSC.

Se tomaron muestras de cultivo de nannochloropsisoceanica diariamente por un tiempo de 10 días, a las cuales se le evaluó: $\mathrm{pH}$, temperatura y conteo celular.

La sanguaza fue calentada a $70^{\circ} \mathrm{C}$ por 20 minutos en un recipiente plástico tapado y luego se sedimentó a $4^{\circ} \mathrm{C}$ por 1 semana, se separó el sobrenadante para formular el medio de cultivo con agua de mar. En las Figuras 1 y 2 se muestran el proceso de tratamiento de la sanguaza a $70^{\circ} \mathrm{C}$ por 20 minutos.

Figura 1. Tratamiento de la sanguaza a $70^{\circ} \mathrm{C} \times 20$ minutos

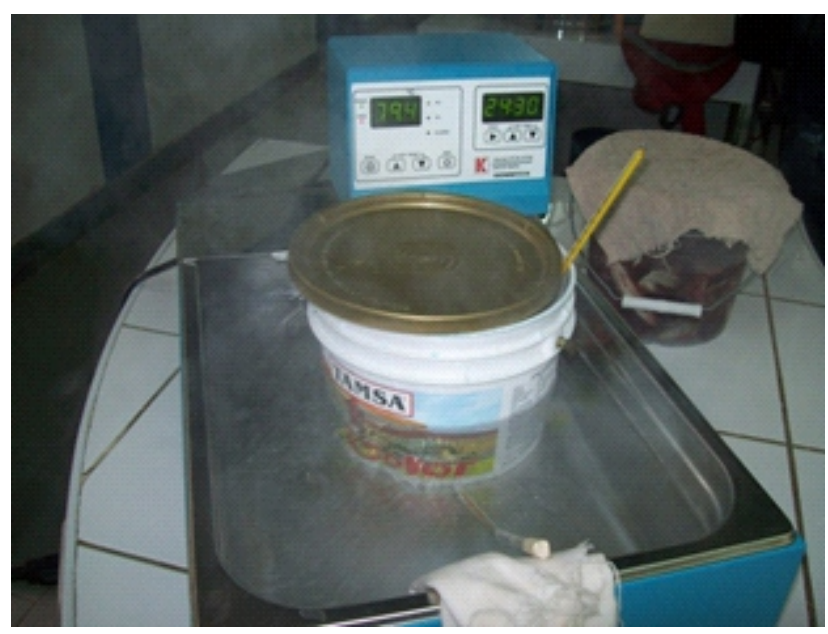

El agua de mar se sedimentó por 10 horas y se filtró al vacío con membrana filtrante de 0,45 um y luego se esterilizó a $105^{\circ} \mathrm{C}$ por 10 minutos. En la Tabla 1 se muestra el análisis fisicoquímico del agua de mar.

Tabla 1. Análisis fisicoquímico del agua de mar

\begin{tabular}{lc}
\hline COMPONENTE & CANTIDAD \\
\hline Temperatura & $22^{\circ} \mathrm{C}$ \\
$\mathrm{pH}$ & $7,5-7,8$ \\
Salinidad & $35,0 \mathrm{ppm}$ \\
$\mathrm{CO}_{2}$ & $15,0 \mathrm{ppm}$ \\
Amonio & $0,05 \mathrm{ppm}$ \\
Nitrito & $0,05 \mathrm{ppm}$ \\
Nitrato & $0,25 \mathrm{ppm}$ \\
Alcalinidad & $120,0 \mathrm{ppm}$ \\
\hline
\end{tabular}

Equipo utilizado: Salt Water, Aquaculture test kit model AQ-4, Code 3635-03 de la Compañía La Motte-USA.

Trabajos Pre-experimentales con cepa microalgal de 
Nannochloropsisoceanica.

Para las pruebas pre-experimentales se formuló el medio de cultivo con sanguaza y agua de mar el cual se reguló a $\mathrm{pH} 7,6$ y luego fue esterilizado a $105^{\circ} \mathrm{C}$ por 10 minutos.

En la tabla 2 se muestra el protocolo pre-experimental que se utilizó para delimitar el efecto de la concentración sanguaza/agua de mar en la producción de biomasa de Nannochloropsisoceanica. La concentración de sanguaza/agua de mar probadas fueron: 2,$5 ; 5,0 ; 10,0 ; y 15,0 \%(\mathrm{v} / \mathrm{v})$, respectivamente.

Tabla 2. Protocolo pre-experimental de la composición de los medios de cultivo para la producción de biomasa de Nannochloropsisoceanica.

\begin{tabular}{cccccc}
\hline \multirow{2}{*}{ COMPONENTES } & \multicolumn{5}{c}{ MEDIO DE CULTIVO } \\
\cline { 2 - 6 } & CONTROL & P1 & P2 & P3 & P4 \\
\hline $\begin{array}{c}\text { SANGUAZA (ml) } \\
\text { AGUA DE MAR } \\
\text { (ml) }\end{array}$ & - & 8,375 & 16,75 & 33,5 & 50,25 \\
MEDIO GUILLARD \\
(ml) \\
INÓCULO \\
$\begin{array}{c}\text { Nannochloropsisocea } \\
\text { nica } \\
\text { (ml) }\end{array}$
\end{tabular}

Los cultivos fueron trabajados a temperatura ambiente y con iluminación constante. La aireación y la dosificación de $\mathrm{CO} 2$ fueron constantes.

La densidad celular se determinó mediante el uso del Hemocitómetro o cámara de Neubauer que es un dispositivo mediante el cual se puede determinar el número de partículas suspendidas en un volumen conocido y de esta manera saber el número en el recipiente original. El recuento se realizó con un microscopio óptico binocular con aumento de 400x.

Se utilizó un pH-metro portátil de mesa debidamente calibrado, para medir el $\mathrm{pH}$ de los cultivos microalgales.

Se utilizó un termómetro de $-10^{\circ} \mathrm{C}$ a $100^{\circ} \mathrm{C}$, para medir la temperatura de los cultivos microalgales y la temperatura ambiente.

\section{RESULTADOS}

Prueba Pre-experimental de la producción de biomasa de Nannochloropsisoceanica utilizando diferentes concentraciones de sanguaza/agua de mar.

La prueba pre-experimental nos ayudó a conocer el comportamiento de la cepa microalgal Nannochloropsisoceanica en el medio heterotrófico sanguaza con agua de mar. Se evaluó la cantidad de células/ml producidas por unidad de tiempo.

En la Figura 3 y Tabla 3 se muestran las condiciones y resultados de la prueba pre-experimental de la producción de biomasa microalgal de Nannochloropsisoceanica.
Figura 3. Foto de la prueba Pre-experimental.

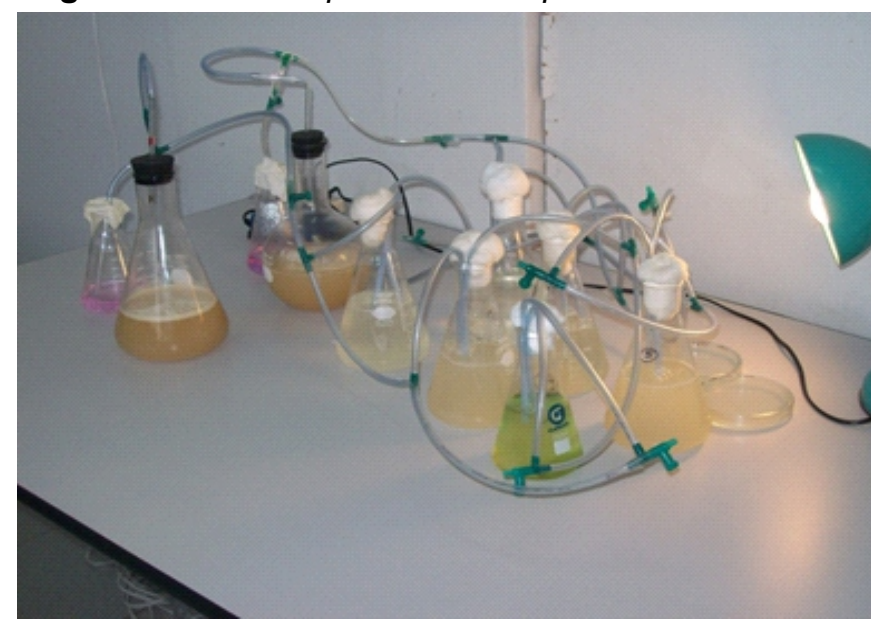

Tabla 3. Valores de la biomasa microalgal producida de Nannochloropsisoceanica en base a la concentración de sanguaza/agua de marprobada.

\begin{tabular}{cccccc}
\hline & \multicolumn{9}{c}{ Células /ml } \\
\cline { 2 - 6 } TIEMPO (hr) & P1: & P2: & P3: & P4: \\
& CONTROL & $\mathbf{2 , 5 \%}$ & $\mathbf{5 , 0 \%}$ & $\mathbf{1 0 , 0 \%}$ & $\mathbf{1 5 , 0 \%}$ \\
& & $\mathbf{v} / \mathbf{v}$ & $\mathbf{v} / \mathbf{v}$ & $\mathbf{v} / \mathbf{v}$ & $\mathbf{v / v}$ \\
\hline 0 & 19 & 19 & 19 & 27 & 16 \\
24 & 27 & 68 & 140 & 265 & 405 \\
96 & 30 & 85 & 513 & 369 & 612 \\
144 & 28 & 80 & 1050 & 1420 & 1050 \\
165 & 32 & 120 & 200 & 390 & 530 \\
191 & 100 & 110 & 230 & 390 & 890 \\
\hline
\end{tabular}

De esta prueba pre-experimental se puede concluir que entre las concentraciones de sanguaza/agua de mar: P2 $(5,0 \% \mathrm{v} / \mathrm{v})$ y P3 $(10,0 \% \mathrm{v} / \mathrm{v})$ se obtuvieron el mayor número de células $/ \mathrm{ml}$ producidas a las 144 horas o sea 6 días.

\section{DISCUSIÓN}

Nuestro resultado del trabajo de investigación utilizando la concentración de sanguaza/agua de mar de 5,0 y $10,0 \%$, se acerca al reportado por Silva (2011) en cuanto al crecimiento máximo de Tetraselmissuecica utilizando una concentración de sanguaza/agua de mar de $4,56 \%$ bajo sus condiciones de trabajo.

De los resultados de este estudio de investigación se demuestra la potencialidad de uso del medio sanguaza/agua de mar en estudios escalables a nivel piloto de producción de biomasa microalgal de Nannochloropsisoceanica, con miras a la producción de biodiesel, debiendose utilizar otro procedimiento de esterilización de la sanguaza para evitar que se precipiten las proteínas y dificultar la recuperación de la biomasa microalgal.

\section{AGRADECIMIENTOS}

A todas aquellas personas que hicieron posible este estudio, de manera especial a los profesionales 
colaboradores: Lic. MoraimaY. Romero C., Ing. Luis A. Huayna P. por sus valiosos aportes en cada una de sus especialidades; a nuestros estudiantes colaboradores: Jean PiereQuiliche D., Estefany Canales C., Selene Herbozo R., Patricia Meneses H., por su apoyo práctico y logístico bibliográfico; así como también al Instituto del Mar del Perú(IMARPE)-Callao por la capacitación en el cultivo de microalgas y al Ing. Héctor Romero Camarena, Jefe del laboratorio Larval de Camarones de la Facultad de Ingeniería Pesquera de la UNJFSC por el obsequio dela cepa de Nannochloropsisoceanica. También se agradece a los laboratorios de Microscopía de la Facultad de Medicina Humana y de Investigación de Bebidas Funcionales de la E.P. de Ing. en Industrias Alimentarias por el apoyo en el préstamo de equipos de laboratorio para la ejecución del proyecto de investigación.

\section{REFERENCIAS BIBLIOGRÁFICAS}

Álvarez, M.; Zarco, J. (1989). Lipids in Microalgae. A review I. Biochemistry. Grasas y Aceites 40(2): 118-145.

Arias, M.; Martínez, A.; Cañizares, R. (2013).

Producción de biodiesel a partir de microalgas: parámetros del cultivo que afectan la producción de lípidos. Acta Biol. Colomb., 18(1), 43-68; recuperado el 26 de Setiembre del 2014, de

www.redalyc.org/pdf/3190/319028010004.pdf

Avagyan A.B. (2008). A contribution to global sustainable development:inclusion of microalgae and their biomass in production and bio cycles. Clean. Tech. Environ. Policy. 10:313-317.

Barbosa, B.; Albrecht, M.;Wijffels, R. (2003).Hydrodynamic stress and lethal events in sparged microalgae cultures.Biotechnol. Bioeng. 83:112-120.

Barbosa, B.;Hadiyanto, M.;Wijffels, R. (2004). Overcoming shear stress of microalgae cultures in spargedphotobioreactors. Biotechnol. Bioeng. 85:78-85.

Bermejo, R.; Álvarez P.; Acien, F.; Molina, G. (2002). Recovery of pure $\mathrm{N}$-phycoerythrin from the microalga Porphyridiumcruentum.Journal of Biotechnology 93: 73-85.

Blas, W.; Rodríguez, J.; Huamán, J.; Vigo, W.; Moya, W.; Sánchez, L.; Pita, A. (2011). Producción de biomasa de Bacillusthuringiensis $\mathrm{H}-14$ var. israelensis mutante en un medio fermentativo a base de sanguaza. REBIOL., $31(2), 8$ págs.

Bold, H.; Wynne, M. (1985). Introduction to the Algae. New Jersey: Prentice Hall. USA.

Chisti Y. (2007). Biodiesel form Microalgae. Biotech Adv. 25: 294-306.

Chisti Y. (2008). Response to Reijnders: Dobiofiels from Microalgae beats biofuels from terrestrial plants. Trends Biotech. 26: 7-7.

Eriksen, N. T. (2008). The technology of microalgal culturing.BiotechnolLett 30:1525-1536.

Garibay, H. A., Vázquez-Duhalt, R., Sánchez, M. P. (2009). Biodiesel a partir de microalgas. Instituto de Biotecnología. Universidad Nacional Autónoma de
México. Cuernavaca, Morelos. BioTecnología, 13(3), 38-61.

González, D.; Marín, M. (2005). Obtención de ensilados biológicos a partir de los desechos del procesamiento de sardinas. RevistaCientífica FCV-LUZ 15(6): 560567.

Guschina, I.; Harwood, J. (2006).Lipids and lipid metabolism in eukaryotic algae. Progress in Lipid Research, 45:160-186.

Herrero, C.; Cid, A.; Fabregas, J.; Abalde, J. (1994). Yields in biomass and chemical constituents of four commercially important marine microalgae with different culture media.Aquacultural Engineering 10: 99-110.

Hirata, K.; Tsujimoto, Y.; Namba, T.; Ohta, T.; Hirayanagi, N.; Miyasaka, H.; Zenk, M.H.; Miyamoto, K. (2001). Strong induction of phytochelatin synthesis by zinc in marine green algae, Dunaliellatertiolecta.Journal of Bioscience and Bioengineering 92: 24-29.

Kalia V.C., Purohit H.J. (2008). Microbial diversity and genomics in aid ofbioenergy.J. Ind. Microbiol. Biotech. 35: 403-419.

Knothe, G.; Dunn, R.; Bagby, M. (1997). Biodiesel: the use of vegetable oilsand their derivatives as alternative diesel fuels. ACS Symp Ser. 666: 172-208.

Lee, Y.K. (1997). Commercial production of microalgae en the Asia-Pacific rim.Journal of Applied Phycology 9: 403-411.

Liu Z.Y., Wang G.C., Zhou B.C. (2008). Effect of iron on growth and lipid accumulation in Chlorella vulgaris.Bioresour. Technol., 99: 4717-22.

Ma F.R., Hanna M.A., (1999). Biodiesel production: a review. BioresourTechnology70:1-15.

Meng X., Yang J., Xu X., Zhang L., Nie Q., Xian M. (2008). Biodiesel production from oleaginous microorganisms.Rev. Ener. 34:1-5.

Miao X., Wu Q. (2004). High yield bio-oil production form fast pyrolysis by metabolic controlling of Chlorella protothecoides. J. Biotech. 110: 85-93.

Molina Grima, E. AcienFernandez, F. G. Garcia Camacho, F. Chisti, Y. (1999).Photobioreactors: light regime, mass transfer, and scale up. J. Biotechnol. 70,231-248.

Mora, R.; Moronta, R.; Ortega, J. Morales, E. (2005). Crecimiento y producción de pigmentos de la microalga nativa Chlorellasp. aislada de la represa de Tulé, Municipio Mara, Estado Zulia, Venezuela. CienciaCompleta: 1-9.

Paerl H.W., Fulton R.S., Moisander P.H. (2001).Harmful freshwater algal blooms, with an emphasis on cyanobacteria. Scientific World Journal 1:76-113.

Rodolfi, L., Zittelli, G. C., Bassi, N., Padovani, G., Biondi, N., Bonini, G., Tredici, M. R. (2009). Microalgae for oil: strain selection, induction of lipid synthesis and outdoor mass cultivation in a low-cost photobioreactor. Biotechnol.Bioeng. 102: 100-112.

Sastre, R. R.; Csogor, Z.; Perner-Nochta, I.; Fleck- 
Schneider, P.; Posten, C.(2007).Scale-down of microalgae cultivations in tubular photo-bioreactors-A conceptualapproach. Journ. Biotechn. 132, 127-133.

Schenk, P. M., Thomas-Hall, S. R., Stephens, E., Marx, U. C., Mussgnug, J. H., Posten, C., Kruse, O. \&Hankamer, B. (2008). Second generation biofuels: high-efficiency microalgae for biodiesel production. Bioenerg. Res. 1:20-43.

Sellner K.G., Doucette G.J., Kirkpatrick G.J. (2003) Harmful algal blooms: causes, impacts and detection. J IndMicrobiolBiotechnol 30:383-406.

Sheehan, J.; Dunahay, T.; Benemann, J.; Roessler, P. (1998). A Look Back at the U.S. Department of Energy's Aquatic Species Program - Biodiesel from Algae. Recuperado el 26 de Setiembre del 2014, de http://www.nrel.gov/docs/legosti/fy98/

Silva, J.; Vásquez, V.; Merino, F. (2011). Producción de biomasa de Tetraselmissuecica empleando agua de mar con sanguaza. Scientia Agropecuaria 2, 13-23.

Simon, N.A.L. Cras; Foulon E. y Lemée R. (2009). Diversity and evolution of marine phytoplankton. C.R. Biologies 332: 159-170.

Song D., Fu J., Shi D. (2008). Exploitation of Oil-Bearing Microalgae for Biodiesel.Chin. Jour. Biotech. 24: 341348.

Van Gerpen J. (2005). Biodiesel processing and production.Fuel. Process. Technol. 86:1097-1107.

Veziroglu TN., Barbir F. (1992). Hydrogen: the wonder fuel. Int. J. Hydrogen Energy. 17:391-404.

Xiaoling, M. \& Wu, Q. (2006). Biodiesel production from heterotrophic microalgal oil.BioresourceTechnology 97(6): 841-846. 